\title{
AVALIAÇÃO DE METAIS TRAÇOS E ÍONS MAJORITÁRIOS EM ÁGUAS DE CHUVA NA CIDADE DE SÃO PAULO
}

\author{
Anna Paula Godoy Fontenele e Jairo J. Pedrotti \\ Departamento de Química, Centro de Ciências e Humanidades, Universidade Presbiteriana Mackenzie, Rua da Consolação, 930 , \\ 01302-907 São Paulo - SP, Brasil \\ Adalgiza Fornaro* \\ Departamento de Ciências Atmosféricas, Instituto de Astronomia, Geofísica e Ciências Atmosféricas, Universidade de São Paulo, \\ Rua do Matão, 1226, 05508-090 São Paulo - SP, Brasil
}

Recebido em 30/1/08; aceito em 22/10/08; publicado na web em 2/4/09

\begin{abstract}
EVALUATION OF TRACE METALS AND MAJOR IONS CONCENTRATIONS IN RAINWATER IN DOWNTOWN SÃO PAULO CITY. Rainfall samples collected in the downtown area of São Paulo city, during 2003, exhibited average concentrations of cadmium, lead and copper of $1.33,8.52$ and $49.5 \mathrm{nmol} \mathrm{L}^{-1}$, respectively. Among the major ions, $\mathrm{NH}_{4}^{+}$was the predominant species followed by $\mathrm{NO}_{3}^{-}$, $\mathrm{SO}_{4}{ }^{2-}$ and $\mathrm{Ca}^{2+}$, with volume weighed mean (VWM) concentrations of 37.1, 20.1, 11.9 and $10.8 \mu \mathrm{mol} \mathrm{L}{ }^{-1}$, respectively. All the determined species showed high inter-events variability, including free $\mathrm{H}^{+}$ions whose VWM concentration was $4.03 \mu \mathrm{mol} \mathrm{L}^{-1}$, corresponding to a $\mathrm{pH}$ value of 5.39 .
\end{abstract}

Keywords: air pollution; trace metals; FIA-stripping detection.

\section{INTRODUÇÃO}

Nas áreas metropolitanas o problema da degradação da qualidade do ar tem-se constituído numa das mais graves ameaças à qualidade de vida de seus habitantes. Na Região Metropolitana de São Paulo (RMSP), um dos maiores aglomerados urbanos do mundo, as fontes de contaminação do ar atmosférico são relacionadas a processos industriais e à intensa frota de veículos leves e pesados. Entretanto, nos últimos anos a queima de combustíveis pela frota veicular tem sido considerada a principal fonte de poluentes atmosféricos. Estimase que em 2003, 1/3 do total de 7,5 milhões de veículos circulavam diariamente na RMSP. Esta frota veicular contribuiu com mais de 90\% das emissões de monóxido de carbono (CO), hidrocarbonetos, óxidos de nitrogênio $\left(\mathrm{NO}\right.$ e $\mathrm{NO}_{2}$ ) e mais de $40 \%$ das emissões de dióxido de enxofre $\left(\mathrm{SO}_{2}\right)$ e material particulado (MP). ${ }^{1}$

Presentes no material particulado, os metais traços, como cádmio, chumbo e cobre, merecem atenção especial devido à elevada toxicidade sobre os organismos, ocasionando efeitos danosos em longo prazo. ${ }^{2}$ A emissão atmosférica destas espécies está amplamente relacionada com processos antrópicos que utilizam altas temperaturas, como queima de combustíveis fósseis, e também devido ao desgaste de peças de veículos. Outras possíveis fontes são incineração de resíduos, fundições, produção de aço e metais não-ferrosos, indústrias de cimento e de pigmentos. ${ }^{2-4}$ Neste sentido, estudos de avaliação do inventário de emissões de poluentes atmosféricos pela frota veicular de São Paulo, a partir de experimentos em túneis, mostraram que em média os veículos são responsáveis pela emissão de $14 \mu \mathrm{g} \mathrm{km}^{-1}$ de chumbo e por $261 \mu \mathrm{g} \mathrm{km}^{-1}$ de cobre. ${ }^{1}$

Historicamente, a utilização do chumbo como aditivo na gasolina resultou em um incremento nas emissões atmosféricas deste metal nos anos 40. Porém, desde o início dos anos 70, tem havido um esforço contínuo para a diminuição do chumbo na gasolina, gerado pelas preocupações relacionadas com os efeitos à saúde humana. Sendo que, no Brasil, o uso do etanol anidro na gasolina permitiu o bani-

*e-mail: fornaro@model.iag.usp.br mento definitivo do chumbo a partir de 1993. Ademais, esta medida possibilitou o início de uso de catalisadores automotivos reduzindo-se assim a emissão de monóxido de carbono, hidrocarbonetos e óxidos de nitrogênio. ${ }^{1,5}$

Outro aspecto a ser considerado em relação aos metais traços é que nos ciclos biogeoquímicos, a atmosfera é um compartimento de suma importância devido ao transporte destas espécies, sendo a deposição atmosférica considerada fonte significativa de metais tóxicos para os ecossistemas naturais, como o solo e as superfícies de lagos e oceanos. Adicionalmente, as características físicas e químicas do aerossol, como tamanho da partícula, pressão de vapor e solubilidade em água, determinarão o processo pelo qual estas espécies serão removidas. Importante observar que $\mathrm{Pb}$ e $\mathrm{Cd}$ se apresentam, predominantemente, na fração fina $(\mathrm{d}<2,5 \mu \mathrm{m})$, que corresponde ao material particulado que atinge as regiões mais profundas do trato respiratório. O cobre encontra-se, geralmente, presente no material particulado com diâmetro aerodinâmico entre 1-5 $\mu \mathrm{m} .^{6,7}$

Por outro lado, a deposição úmida retira da atmosfera o material particulado e, para certos metais traços, este mecanismo é de extrema importância para muitos ecossistemas. Fenômenos como a acidificação dos solos, das superfícies aquáticas e de lençóis freáticos ocasionados pela "chuva ácida" podem influenciar como estas espécies são distribuídas e acumuladas através dos compartimentos de um ecossistema. $\mathrm{O} \mathrm{pH}$ da água de chuva pode influenciar na solubilidade de compostos contendo metais traços. Vale ressaltar que em águas naturais a solubilidade dos metais segue a ordem: $\mathrm{Cd}>\mathrm{Cu}>\mathrm{Pb}$, sendo esta fração solúvel biodisponível para interagir com os seres vivos. ${ }^{5,8-10}$

Na cidade de São Paulo, vários estudos têm sido realizados na fase líquida da atmosfera envolvendo a avaliação de cátions e ânions majoritários, ${ }^{11,12}$ ácidos carboxílicos, ${ }^{13}$ aldeídos de cadeia curta ${ }^{14} \mathrm{e}$ $\mathrm{H}_{2} \mathrm{O}_{2},{ }^{15}$ enquanto que os metais de transição $(\mathrm{Cu}, \mathrm{Fe}, \mathrm{Mn}, \mathrm{Zn}$ e Ni, entre outros) têm sido avaliados em material particulado coletado em algumas regiões da cidade. ${ }^{4}$ Já em águas de chuva, os primeiros resultados sobre a determinação de metais referem-se à avaliação de $\mathrm{Pb}^{2+}$ em amostras coletadas no centro da cidade do período de janeiro e maio de $2000 .^{16}$ 
Neste trabalho apresentam-se os resultados das determinações de cobre, chumbo e cádmio bem como de cátions e ânions majoritários em águas de chuvas coletadas na região central de São Paulo. Técnicas estatísticas de análise multivariadas foram aplicadas com o objetivo de avaliar possíveis relações entre as concentrações dos componentes iônicos majoritários $\left(\mathrm{NH}_{4}^{+}, \mathrm{Na}^{+}, \mathrm{K}^{+}, \mathrm{Ca}^{2+}, \mathrm{Mg}^{2+}, \mathrm{Cl}^{-}, \mathrm{NO}_{3}^{-}, \mathrm{SO}_{4}^{2-}\right.$, $\mathrm{HCOO}^{-}$e $\mathrm{F}^{-}+\mathrm{CH}_{3} \mathrm{COO}^{-}+\mathrm{HOCH}_{2} \mathrm{COO}^{-}$) e dos metais traços.

\section{PARTE EXPERIMETAL}

\section{Região do estudo}

A cidade de São Paulo juntamente com 38 municípios vizinhos forma a Região Metropolitana de São Paulo, RMSP, localizada a $23^{\circ} \mathrm{S}$ e $46^{\circ} \mathrm{W}$ na porção sudeste do Brasil. Possui área de $8.051 \mathrm{~km}^{2}$ e população superior a 17 milhões de habitantes, distribuída de forma desordenada em uma área urbanizada de $1.747 \mathrm{~km}^{2}$, consistindo na região mais industrializada e populosa da América Latina. Em termos de precipitação, o período chuvoso entre dezembro e fevereiro e o período de seca entre junho e agosto são bem definidos - meses correspondentes ao verão e inverno, respectivamente. Situações intermediárias ocorrem no outono e primavera. ${ }^{11}$

\section{Amostragem}

As amostragens de água de chuva foram realizadas com coletor automático para deposição úmida apenas, modelo G. K. Walter, instalado no topo de um prédio do campus da Universidade Mackenzie ( $\sim 15 \mathrm{~m}$ de altura), entre janeiro e dezembro de 2003. O ponto de amostragem localiza-se na região central da cidade de São Paulo ( $\left.23^{\circ} 32^{\prime} \mathrm{S}, 46^{\circ} 39^{\prime} \mathrm{W}\right)$, situado próximo à Rua da Consolação $(80 \mathrm{~m})$, um dos pontos mais trafegados da cidade com intensa circulação de veículos leves, pesados (ônibus, principalmente) e pedestres. A abertura do funil do amostrador, posicionado a $1,5 \mathrm{~m}$ do piso, tem área de captação de $500 \mathrm{~cm}^{2}$. No interior do gabinete do amostrador, protegido das intempéries, fica alojado frasco coletor de $5,5 \mathrm{~L}$ de capacidade. Os frascos de polietileno de alta densidade destinados à armazenagem das amostras foram tratados conforme procedimento descrito anteriormente. ${ }^{12}$

Logo após o término do evento de precipitação, ou pela manhã (para eventos noturnos), os frascos foram transferidos para o labora- tório e foram separadas alíquotas para as diferentes determinações, mantendo-se as frações destinadas às medidas de $\mathrm{pH}$ e metais traços sob refrigeração $\left(-4^{\circ} \mathrm{C}\right)$ e frações filtradas em membranas de policarbonato de $0,22 \mu \mathrm{m}$ de porosidade que foram congeladas $\left(-15^{\circ} \mathrm{C}\right)$ para as análises cromatográficas. As medidas de $\mathrm{pH}$ foram realizadas em temperatura controlada $\left(25 \pm 1{ }^{\circ} \mathrm{C}\right)$, no máximo 10 dias após a refrigeração. As amostras para análise de metais traços ainda foram condicionadas em meio de solução de $\mathrm{HNO}_{3} 0,1 \%$.

\section{Soluções e reagentes}

Todas as soluções foram preparadas a partir de reagentes de grau analítico (Merck) e empregando-se água desionizada (resistividade $>18 \mathrm{M} \Omega \mathrm{cm}^{-1}$ ) em sistema de purificação Nanopure ${ }^{\circledR}$.

O ácido nítrico utilizado como eletrólito de suporte, na preparação das soluções de referência de $\mathrm{Cd}^{2+}, \mathrm{Pb}^{2+}$ e $\mathrm{Cu}^{2+}$ e na acidificação das amostras para a análise destas espécies foi purificado por destilação a sub-ebulição usando destilador de quartzo Marconi Equipamentos de Laboratório, modelo 075.

\section{Instrumentação e metodologia analítica}

Mediu-se o pH com eletrodo de vidro combinado com Ag/ $\mathrm{AgCl}$ ( $\mathrm{KCl}$ saturado) conectado a potenciômetro Digimed DM-20. Os íons majoritários foram determinados por cromatografia iônica (cromatógrafo Metrohm modelo 761) com detecção condutométrica. $\mathrm{Na}$ análise dos ânions, empregou-se solução eluente de $\mathrm{NaHCO}_{3}$ $1,7 \mathrm{mmol} \mathrm{L}{ }^{-1} / \mathrm{Na}_{2} \mathrm{CO}_{3} 1,8 \mathrm{mmol} \mathrm{L}^{-1}$, vazão de $1 \mathrm{~mL} \mathrm{~min}^{-1}$, alça de amostragem de $20 \mu \mathrm{L}$, coluna Metrosep A-Supp 5, coluna supressora Metrohm e, como regenerante, solução aquosa de $\mathrm{H}_{2} \mathrm{SO}_{4} 50$ mmol L-1. Na determinação dos cátions, utilizou-se como solução eluente ácido dipicolínico $0,75 \mathrm{mmol} \mathrm{L}^{-1} /$ ácido tartárico 4 mmol L-1 e $5 \%$ de acetona, vazão de $1 \mathrm{~mL} \mathrm{~min}^{-1}$, alça de amostragem de 100 $\mu \mathrm{L}$ e coluna Metrosep C2 250.

Cádmio, chumbo e cobre foram determinados usando sistema de análise por injeção em fluxo (FIA) com detecção por voltametria de redissolução anódica, conforme ilustra a Figura 1. Uma bomba peristáltica Ismatec Reglo foi usada para impulsionar o fluido transportador (solução de $\mathrm{HNO}_{3} 10 \mathrm{mmol} \mathrm{L}^{-1}$ ) sob vazão $0,5 \mathrm{~mL} \mathrm{~min}^{-1}$. Um injetor manual, confeccionado com válvulas solenóides, provido com alça de amostragem de $500 \mu \mathrm{L}$ foi usado para introduzir as amostras e

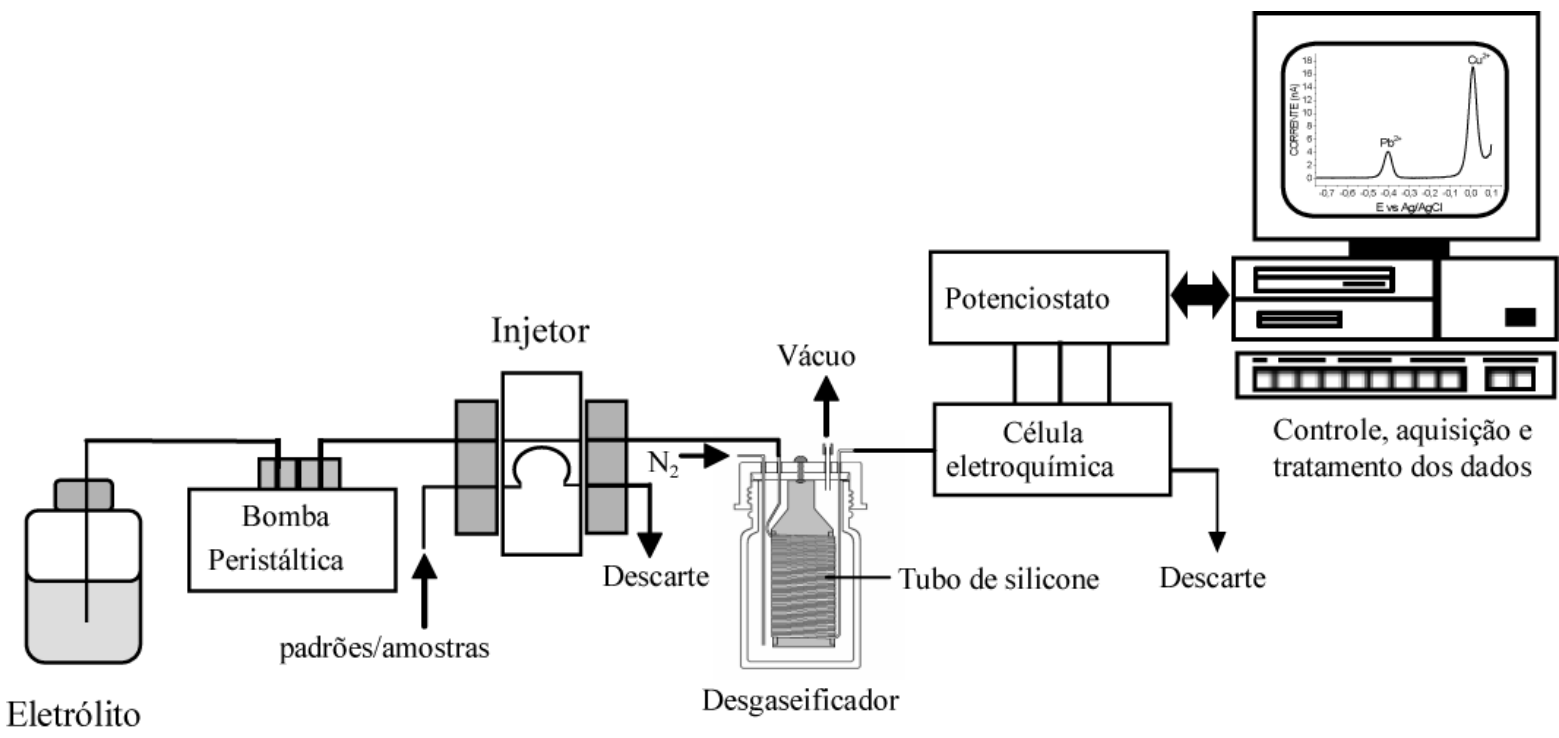

Figura 1. Diagrama esquemático do sistema de análise por injeção em fluxo (FIA) com detecção por voltametria de redissolução anódica 
soluções analíticas no percurso analítico. Para remover a interferência do oxigênio dissolvido nas soluções utilizou-se um desgaseificador contínuo que utiliza tubo de silicone de $0,5 \mathrm{~mm}$ de d.i. mantido em atmosfera inerte sob pressão reduzida, inserido entre o injetor e a célula eletroquímica. ${ }^{17}$ Os experimentos amperométricos e voltamétricos foram feitos com potenciostato ( $\mu$ Autolab type II, Ecochemie) combinado com o módulo de eletrodos (VA Stand 663, Metrohm) constituído de eletrodo de referência de $\mathrm{Ag} / \mathrm{AgCl}$, do eletrodo de gota pendente de mercúrio (eletrodo de trabalho) e do eletrodo auxiliar de carbono vítreo. A adaptação da célula eletroquímica convencional para o sistema em fluxo foi feita através da inserção de um adaptador de PTFE em formato de "L", posicionado na extremidade do capilar de vidro e bem próximo à gota de $\mathrm{Hg} .{ }^{18}$ Estas medições foram realizadas à temperatura controlada $\left(25 \pm 1{ }^{\circ} \mathrm{C}\right)$

\section{Procedimento para determinação de metais}

Para aumentar a sensibilidade na detecção dos metais, o módulo de eletrodo de gota de mercúrio foi programado para gerar gotas de maior tamanho, área igual a $0,503 \mathrm{~mm}^{2}$. Sob vazão do fluido transportador de $0,5 \mathrm{~mL} \mathrm{~min}^{-1}$, o tempo gasto para a solução amostra ou padrão alcançar o detector é de $75 \mathrm{~s}$. Assim, imediatamente após a injeção da solução no percurso analítico, o potencial do eletrodo de $\mathrm{Hg}$ foi mantido em $0,0 \mathrm{~V}$ durante $70 \mathrm{~s}$. Após esta etapa, o potencial de $-0,75 \mathrm{~V}$ foi aplicado ao eletrodo de trabalho durante $120 \mathrm{~s}$ para eletrodeposição de $\mathrm{Cd}^{2+}, \mathrm{Pb}^{2+}$ e $\mathrm{Cu}^{2+}$ (etapa de pré-concentração). O sincronismo entre injeção da amostra e o início da etapa de pré-concentração foi estabelecido com base no registro de curvas amperométricas de $\mathrm{Cd}(\mathrm{II}) 1 \times 10^{-5} \mathrm{~mol} \mathrm{~L}^{-1}$. A largura do pico junto à linha base é de $110 \mathrm{~s}$. Assim, a pré-concentração no período de 70 a 190 s engloba o pico com margem de tolerância de alguns segundos e reduz exposição desnecessária do eletrólito ao potencial de coleta do material eletroativo. Após este período, foi feita a varredura de pulso diferencial entre $-0,75$ e $0,15 \mathrm{~V}$ sob velocidade de $10 \mathrm{mV} \mathrm{s}^{-1}$ e amplitude de pulso de $50 \mathrm{mV}$. A quantificação dos metais foi feita através do método de adição de padrão. Os limites de detecção, estimados através do método dos mínimos quadrados das curvas de calibração (LD = sinal do "branco" (coeficiente linear) +3 x desvio padrão do "branco", $\left.s_{x / y}\right),{ }^{19}$ para cobre, chumbo e cádmio foram de 0,50; 0,40 e $0,010 \mu \mathrm{g} \mathrm{L}^{-1}$, respectivamente.

\section{Tratamento de dados}

O software Statistica 7.0. foi utilizado para o tratamento dos dados através da análise de fatores (AF) e análise de cluster (AC).

A AF é um método estatístico atrativo e amplamente aplicado a amostras atmosféricas com o objetivo de identificação de fontes poluidoras. Esta ferramenta permite trabalhar com grupo grande de variáveis com unidades distintas e ordens de grandeza bastante díspares, agrupando-as sob uma mesma "tendência", não necessitando, a priori, do conhecimento do número e da composição das fontes de poluentes.

Para que os resultados da aplicação da AF sejam estatisticamente significativos é necessário, como condição mínima, um número considerável de amostras em relação ao número de variáveis:

$\mathrm{N} \geq 30+\frac{\mathrm{n}+3}{2}$

onde, $\mathrm{N}$ é o número total de amostras e $\mathrm{n}$ é o número de variáveis.

No presente estudo, o conjunto de 44 amostras de águas de chuva foi empregado como matriz. As variáveis avaliadas $(n=14)$, além dos cátions e ânions majoritários, foram os íons cádmio, chumbo e cobre.
$\mathrm{Na}$ AC deve-se, inicialmente, desenvolver uma escala quantitativa para medir a associação (similaridade ou dissimilaridade) entre os objetos para depois agrupá-los. O resultado é apresentado em uma árvore hierárquica (dendograma). A seguir, calculam-se as distâncias entre os objetos e aplicam-se algoritmos para encontrar clusters mais adequados. Neste estudo, foi empregado o método de Ward, para cálculo de distâncias entre os clusters, a partir do cálculo das distâncias entre as variáveis pela correlação de Pearson. ${ }^{20}$

\section{RESULTADOS E DISCUSSÃO}

O procedimento analítico de análise por injeção em fluxo proporciona excelente sensibilidade e consumo reduzido de amostra para determinação simultânea dos metais em estudo no intervalo de concentração que se estende de 10 a $500 \mathrm{nmol} \mathrm{L}^{-1}$. A freqüência analítica atinge 12 determinações/hora sob vazão de $0,5 \mathrm{ml} \mathrm{min}^{-1}$ do fluido transportador e tempo de pré-concentração de 120 s. A reprodutibilidade das medições do sistema analítico foi avaliada através da injeção sucessiva de $500 \mu \mathrm{L}$ de uma solução de $\mathrm{Pb}$ (II) $1 \times 10^{-7} \mathrm{~mol} \mathrm{~L}^{-1}$ (4 ng). Os sinais de corrente de pico de uma série de 20 voltamogramas de redissolução apresentaram sinal médio de 25,0 nA, dentro do intervalo de 24,8-25,4 nA. Isto corresponde a um desvio padrão relativo, na concentração de $20 \mathrm{ppb}$, inferior a $1 \%$.

Os resultados da avaliação da composição iônica majoritária e metais traços em águas de chuva estão apresentados na Tabela 1. Neste conjunto de amostras, observou-se grande variabilidade na concentração das diferentes espécies analisadas. O íon predominante foi amônio, seguido de nitrato e cálcio. Os ânions fluoreto, acetato e glicolato apresentaram mesmo tempo de retenção na condição analítica em que foram determinadas as concentrações aniônicas nas

Tabela 1. Concentrações médias ponderadas pelo volume (MPV); médias aritméticas, desvio padrão (dp), valores mínimos e máximos das concentrações dos metais traços e dos íons majoritários em águas de chuva $(\mathrm{N}=44)$ da cidade de São Paulo, do período entre janeiro e dezembro de 2003

\begin{tabular}{|c|c|c|c|c|}
\hline & mínimo & máximo & Média $( \pm d p)$ & MPV \\
\hline & \multicolumn{4}{|c|}{ nmol L-1 } \\
\hline $\mathrm{Cd}^{2+}$ & 0,60 & 5,2 & $1,33(1,20)$ & 1,21 \\
\hline $\mathrm{Pb}^{2+}$ & 2,0 & 45 & $8,52(8,30)$ & 7,86 \\
\hline \multirow[t]{2}{*}{$\mathrm{Cu}^{2+}$} & 2,1 & 243 & $49,5(53,0)$ & 41,7 \\
\hline & \multicolumn{4}{|c|}{$\mu \mathrm{mol} \mathrm{L}{ }^{-1}$} \\
\hline $\mathrm{H}^{+}$ & 0,074 & 16,6 & $2,20(3,70)$ & 4,03 \\
\hline $\mathrm{Na}^{+}$ & 0,79 & 52,3 & $10,6(10,6)$ & 8,64 \\
\hline $\mathrm{NH}_{4}^{+}$ & 3,65 & 122 & $41,0(27,5)$ & 37,1 \\
\hline $\mathrm{K}^{+}$ & 0,45 & 66,5 & $8,49(11,7)$ & 9,55 \\
\hline $\mathrm{Ca}^{2+}$ & 1,30 & 47,4 & $12,7(10,1)$ & 10,8 \\
\hline $\mathrm{Mg}^{2+}$ & 1,66 & 12,2 & $3,29(2,20)$ & 3,30 \\
\hline $\mathrm{A}^{-*}$ & 3,62 & 88,0 & $27,3(20,2)$ & 22,5 \\
\hline For & 0,23 & 72,3 & $7,17(14,4)$ & 4,79 \\
\hline $\mathrm{Cl}^{-}$ & 1,4 & 34,9 & $9,85(6,71)$ & 9,29 \\
\hline $\mathrm{NO}_{3}^{-}$ & 2,55 & 75,6 & $18,0(15,0)$ & 20,1 \\
\hline $\mathrm{SO}_{4}^{2-}$ & 1,81 & 38,9 & $11,6(9,00)$ & 11,9 \\
\hline
\end{tabular}

$\mathrm{A}^{*}$ refere-se à soma dos ânions acetato, glicolato e fluoreto que coeluem na condição analítica do presente estudo. 
amostras de águas de chuva deste estudo. Devido à soma das contribuições destes três íons resultar em concentração alta, relativamente às outras espécies estudadas, optou-se por considerá-las como um único componente nas análises a seguir.

No ano de 2003 a quantidade de chuva mensal foi em geral $30 \%$ menor que a pluviometria média mensal da série histórica (1973-2003). ${ }^{13}$ Comparando-se com dados de MPV de período mais longo, entre julho de 2002 e junho de 2004, onde foi possível avaliar o efeito das estações seca e chuvosa, os resultados apresentados na Tabela 1 mostram valores muito próximos às MPV do período seco, principalmente, para amônio e cálcio. ${ }^{12}$

Entre os metais traços, cobre foi a espécie predominante com concentração média de $3,14 \mu \mathrm{g} \mathrm{L^{-1 }}$, enquanto cádmio apresentou as concentrações mais baixas, valor médio de $0,15 \mu \mathrm{g} \mathrm{L}^{-1}$. Vale ressaltar que as concentrações mais elevadas foram observadas em amostras coletadas após vários dias sem ocorrência de eventos de chuva, sugerindo acumulação dos poluentes na atmosfera durante o período seco. A Tabela 1 mostra grande variabilidade no intervalo de concentrações e no desvio padrão, o que pode estar relacionado a vários fatores, como alterações meteorológicas e processos locais de emissão. ${ }^{21,22}$

Apesar de haver poucos trabalhos na literatura sobre determinação de metais traços em águas de chuva foi possível comparar-se os resultados deste trabalho com os de outras áreas urbanas (Figura 2); como Singapura, capital do país de mesmo nome, ${ }^{9}$ Paris (França), ${ }^{21}$ Göttingen (Alemanha), ${ }^{23}$ Paradise (Nova Zelândia), ${ }^{24}$ Ajlune (Jordânia), ${ }^{25}$ New Castle (Estados Unidos) ${ }^{26}$ e Atenas (Grécia) ${ }^{27}$. A comparação com São Paulo corrobora a complexidade associada à presença destes metais em águas de chuva de regiões urbanas. Excetuando-se Gottingen, em todas as outras cidades, o período de amostragem correspondeu a alguns anos após o banimento do uso de chumbo como aditivo na gasolina.

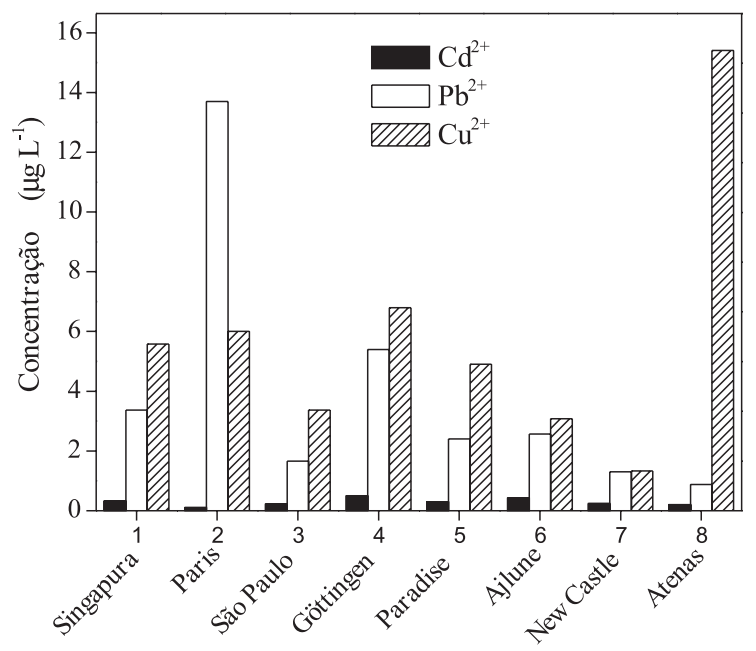

Figura 2. Comparação entre valores da concentração média $\left(\mu g L^{-1}\right)$ de cádmio, chumbo e cobre em águas de chuva de São Paulo (presente estudo) e outras cidades ${ }^{9,21,23-27}$

Em Singapura, as emissões de $\mathrm{Cd}$ e $\mathrm{Pb}$ foram associadas, predominantemente, aos incineradores de lixo urbano, uma vez que óxidos destes metais também estão presentes em pigmentos, estabilizadores e catalisadores de processos poliméricos. O cobre foi correlacionado com os processos que utilizam altas temperaturas, principalmente queima de combustíveis fósseis. ${ }^{9}$

Em Paris, a presença dos metais em águas de chuva foi considerada, predominantemente, de emissões urbanas. Na Figura 2 observa-se que Paris apresentou a mais alta concentração de chumbo, apesar do período do estudo ter sido realizado 7 anos após o banimento do uso de chumbo na gasolina, com a observação dos autores de que na década de 80 as concentrações de chumbo foram quatro vezes mais altas, atingindo $60 \mu \mathrm{g} \mathrm{L}^{-1}$.

Göttingen é uma cidade de porte médio e está localizada próxima a sítios urbano-industriais alemães. As concentrações de chumbo na Alemanha foram reduzidas em 95\% entre 1981-1995 devido a filtros instalados nas indústrias e início da utilização da gasolina isenta de chumbo. ${ }^{23}$

Paradise é uma região situada na ilha sul da Nova Zelândia, apesar de ser considerada não impactada, suas águas de chuva apresentaram concentrações relativamente significativas de chumbo e cobre. ${ }^{24}$ Ajlune é uma cidade pequena, localizada a $80 \mathrm{~km}$ de Amman, capital da Jordânia. As regiões que circundam esta cidade são essencialmente rurais, não havendo qualquer influência direta de fontes antropogênicas. ${ }^{25} \mathrm{Em}$ New Castle, as coletas de águas de chuva foram realizadas em uma região costeira, situada a jusante da região industrializada do vale do rio Ohio. ${ }^{26} \mathrm{O}$ ponto de amostragem em Atenas localizou-se próximo à região altamente industrializada, com intensa emissão de cobre. ${ }^{27}$

A variabilidade das concentrações de metais em águas de chuva observadas em regiões que não são grandes centros urbano-industriais, mas que se localizam próximas a estes, foram relacionadas com a direção do vento. ${ }^{9,21,24-27}$

Nas águas de chuva de São Paulo verificou-se que as concentrações para chumbo e cobre foram menores que aquelas encontradas em outras regiões urbanas e industrializadas como Paris, Singapura e Göttingen, no entanto, foram maiores que em New Castle.

Estudos sobre a determinação de metais pesados em águas de chuva no Brasil são raros. Avaliação da literatura permitiu comparar os resultados para cobre e chumbo. Em estudo realizado em Florianópolis (Santa Catarina), determinaram-se concentrações médias aritméticas de cobre em $6,3 \mathrm{nmol} \mathrm{L}^{-1}\left(0,40 \mu \mathrm{g} \mathrm{L}^{-1}\right)$, sendo o valor máximo de $37,8 \mathrm{nmol} \mathrm{L}-1$ (2,4 $\left.\mu \mathrm{g} \mathrm{L}^{-1}\right) .{ }^{28}$ No Rio Grande do Sul, em Candiota sob influência de termelétrica a carvão, a quantidade média de cobre foi de $30,7 \mathrm{nmol} \mathrm{L}^{-1}\left(1,95 \mu \mathrm{g} \mathrm{L}^{-1}\right)$, enquanto que na cidade de Porto Alegre a concentração média de cobre foi de 43,5 $\mathrm{nmol} \mathrm{L}^{-1}$ $\left(2,76 \mu \mathrm{g} \mathrm{L}^{-1}\right) .{ }^{29}$ Portanto, comparando-se com estas diferentes regiões do Brasil a concentração média de cobre, 49,5 nmol L-1 $(3,14 \mu \mathrm{g}$ $\left.\mathrm{L}^{-1}\right)$, em águas de chuva de São Paulo foi mais elevada. Em relação à contaminação por chumbo, as águas de chuva avaliadas neste estudo apresentaram concentração comparável a estudo anterior, realizado no mesmo ponto de amostragem, entre janeiro e maio de $2000 .^{16}$

\section{Análise estatística}

Complementando-se os estudos de avaliação da composição química de águas de chuva em São Paulo que vêm sendo realizados, de forma inédita conseguiu-se no presente trabalho determinar, em conjunto com a composição iônica majoritária, a concentração dos metais $\mathrm{Cd}, \mathrm{Pb}$ e $\mathrm{Cu}$, ou seja, um total de 14 variáveis em 44 amostras de águas de chuva o que possibilitou a aplicação da análise estatística multivariada (análise de fatores e de clusters).

Após a rotação varimax, obtiveram-se quatro fatores (autovalor > 1), assim como os pesos e as comunalidades apresentadas na Tabela 2. Estes quatro fatores explicaram, respectivamente, $37,13,11$ e $10 \%$ da variância, ou seja, explicaram o total de $71 \%$ da variância.

Nos resultados da AF, o fator 1 agrupou as espécies $\mathrm{NH}_{4}^{+}, \mathrm{SO}_{4}^{2-}$, $\mathrm{Ca}^{2+}, \mathrm{Cl}^{-}$e HCOO- O amônio tem sido a espécie predominante entre os íons solúveis majoritários nas águas de chuva de São Paulo. ${ }^{12} \mathrm{O}$ amônio na atmosfera é originalmente emitido como amônia $\left(\mathrm{NH}_{3}\right)$, sendo que sua emissão predominante tem sido associada a fontes biogênicas (esgoto doméstico, emissões de animais etc). ${ }^{30}$ Entretanto, 
Tabela 2. Pesos dos fatores, comunalidades (h2) e variâncias extraídas da análise de fatores para metais-pesados e íons majoritários em amostras de águas de chuva da região central de São Paulo do ano de 2003

\begin{tabular}{lccccc}
\hline Variáveis & Fator 1 & Fator 2 & Fator 3 & Fator 4 & h2 \\
\hline $\mathrm{Cd}^{2+}$ & 0,02 & 0,12 & 0,31 & $\mathbf{0 , 8 2}$ & 0,70 \\
$\mathrm{~Pb}^{2+}$ & 0,12 & 0,52 & $-0,17$ & $\mathbf{0 , 7 0}$ & 0,81 \\
$\mathrm{Cu}^{2+}$ & 0,28 & 0,04 & 0,05 & $\mathbf{0 , 8 6}$ & 0,77 \\
$\mathrm{H}^{+}$ & $-0,24$ & $\mathbf{0 , 7 7}$ & 0,03 & $-0,04$ & 0,63 \\
$\mathrm{Na}^{+}$ & 0,34 & $\mathbf{0 , 7 0}$ & 0,16 & 0,08 & 0,77 \\
$\mathrm{NH}_{4}^{+}$ & $\mathbf{0 , 8 1}$ & $-0,02$ & $-0,08$ & 0,29 & 0,82 \\
$\mathrm{~K}^{+}$ & 0,34 & $\mathbf{0 , 7 3}$ & 0,13 & 0,28 & 0,79 \\
$\mathrm{Ca}^{2+}$ & $\mathbf{0 , 8 0}$ & 0,28 & 0,05 & 0,16 & 0,81 \\
$\mathrm{Mg}^{2+}$ & $-0,03$ & $-0,09$ & $\mathbf{0 , 8 5}$ & 0,12 & 0,48 \\
$\mathrm{~A}^{-}$ & 0,28 & 0,03 & $\mathbf{0 , 8 6}$ & 0,07 & 0,72 \\
$\mathrm{For}^{-}$ & $\mathbf{0 , 8 4}$ & 0,04 & 0,10 & 0,32 & 0,63 \\
$\mathrm{Cl}^{-}$ & $\mathbf{0 , 8 1}$ & 0,11 & 0,43 & $-0,04$ & 0,88 \\
$\mathrm{NO}_{3}^{-}$ & 0,46 & $\mathbf{0 , 6 8}$ & $-0,08$ & 0,28 & 0,80 \\
SO $_{4}^{2-}$ & $\mathbf{0 , 7 4}$ & 0,45 & 0,13 & 0,25 & 0,92 \\
\hline Autovalores & $\mathbf{5 , 7}$ & $\mathbf{2 , 0}$ & $\mathbf{1 , 6}$ & $\mathbf{1 , 4}$ & \\
\hline Variância total $\mathbf{( \% )}$ & $\mathbf{3 7}$ & $\mathbf{1 3}$ & $\mathbf{1 1}$ & $\mathbf{1 0}$ & $\mathbf{7 1}$ \\
\hline
\end{tabular}

outra importante contribuição para a amônia/amônio na atmosfera de São Paulo pode ser devida processos que ocorrem nos catalisadores de três vias, usados nos veículos produzidos no Brasil. Alguns estudos mostram que nestes catalisadores, a redução dos óxidos de nitrogênio não tem formado apenas $\mathrm{N}_{2}$, mas também amônia, variando entre $1 \mathrm{e}$ $170 \mathrm{mg} \mathrm{km}{ }^{-1} \cdot{ }^{31} \mathrm{~A}$ presença dos íons sulfato e cálcio neste fator podem estar associados com a solubilização de partículas provenientes de atividades da construção civil. ${ }^{4}$

Destaca-se também o fator 4, que agrupou os metais cádmio, cobre e chumbo, cuja presença em águas de chuva pode ser devida à solubilização de partículas atmosféricas contendo estes metais. Sendo estas provenientes, predominantemente, das emissões veiculares em função do desgaste de diferentes componentes dos veículos, além do sistema de exaustão, devido à presença destas espécies tanto no álcool quanto no diesel. ${ }^{32}$

Os resultados da análise de fatores indicaram características peculiares entre as diferentes espécies iônicas presentes na atmosfera da RMSP, sendo que a análise de cluster se mostrou como ferramenta complementar à análise de fatores. O dendograma da Figura 3 separa dois grandes grupos, sendo o primeiro constituído das espécies A; $\mathrm{Mg}^{2+}, \mathrm{Cl}^{-}, \mathrm{SO}_{4}^{2-}, \mathrm{Ca}^{2+}$, For, $\mathrm{NH}_{4}^{+}$e no segundo destaca-se o agrupa-

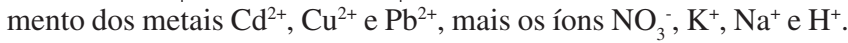
Pode-se considerar o primeiro grupo como efeito da urbanização e processos industriais em geral, responsáveis por parte da emissão de compostos de enxofre presentes na atmosfera de São Paulo, ${ }^{1}$ enquanto que o segundo grupo pode estar indicando a contribuição das emissões veiculares, responsáveis por $95 \%$ da emissão dos óxidos de nitrogênio $\left(\mathrm{NO}_{\mathrm{x}}=\mathrm{NO}+\mathrm{NO}_{2}\right),{ }^{1}$ sendo o íon $\mathrm{NO}_{3}{ }^{-}$um dos principais produtos da oxidação destes compostos. ${ }^{2}$

\section{CONCLUSÕES}

Determinações de $\mathrm{Cd}^{2+}, \mathrm{Pb}^{2+}$ e $\mathrm{Cu}^{2+}$ e de íons majoritários foram realizadas em águas de chuvas coletadas no centro da cidade de São

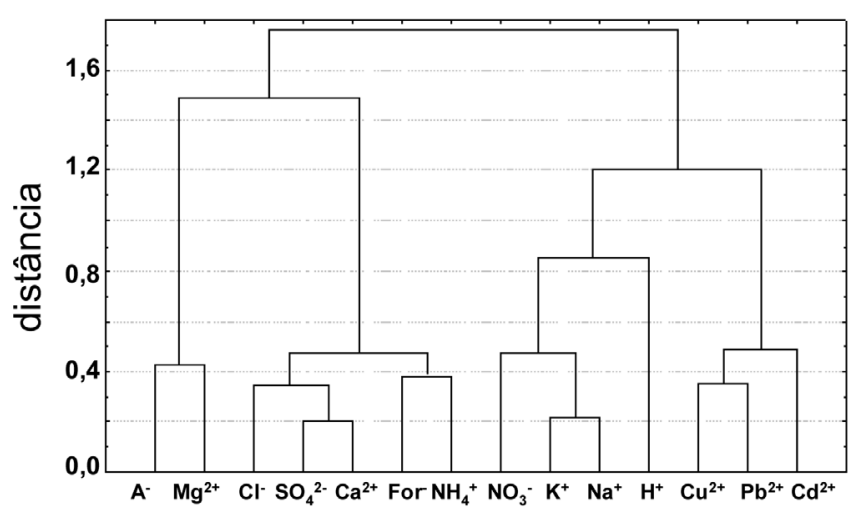

Figura 3. Dendograma para 14 espécies iônicas determinadas em águas de chuva da cidade de São Paulo empregando o método de Ward, com distâncias calculadas segundo a correlação de Pearson entre as variáveis

Paulo entre janeiro e dezembro de 2003. A avaliação das concentrações dos metais traços mostrou predominância de $\mathrm{Cu}^{2+}$, seguido de $\mathrm{Pb}^{2+} \mathrm{e} \mathrm{Cd}^{2+}$. No período deste estudo, a pluviometria média foi abaixo da normal histórica. A composição iônica majoritária apresentou valores de concentração média ponderada pelo volume característica de períodos secos. $\mathrm{NH}_{4}^{+}$foi a espécie iônica predominante, seguida de $\mathrm{NO}_{3}{ }^{-} \mathrm{e} \mathrm{Ca}^{2+}$. $\mathrm{O}$ valor de $\mathrm{pH}$ das amostras variou entre 4,78 e 7,13 e MPV $=5,39$, indicando que as águas de chuva no período foram levemente ácidas.

Para a avaliação da contaminação das águas de chuva da RMSP, as aplicações da análise fatorial e de cluster mostraram-se ferramentas estatísticas complementares importantes, porém também indicaram a complexidade envolvida na interpretação dos dados de composição química nos processos atmosféricos.

\section{AGRADECIMENTOS}

Ao CNPq e à FAPESP pelo suporte financeiro e ao Dr. O. S. Ccoyllo pelo auxílio no uso do software Statistica 7.0 e discussões dos dados obtidos. Os autores agradecem também aos anônimos assessores pelas críticas e sugestões que contribuíram para a melhoria do manuscrito.

\section{REFERÊNCIAS}

1. CETESB - Companhia de Tecnologia de Saneamento Ambiental; Relatório de Qualidade do Ar no Estado de São Paulo - 2003, Secretaria do Meio Ambiente, Série Relatórios, ISSN 0103-4103, São Paulo, 2004; http://www.cetesb.sp.gov. br, acessada em Setembro 2004; Martins, L. D.; Andrade, M. F.; Freitas, E. D.; Pretto, A.; Gatti, L. V.; Albuquerque, E. L.; Tomaz, E.; Guardani, M. L.; Martins, M. H. R. B.; Junior, O. M. A.; Environ. Sci. Technol. 2006, 40, 6722; SanchezCcoyllo, O. R.; Ynoue, R. Y.; Martins, L. O.; Astolfo, R.; Miranda, R. M.; Freitas, E. D.; Borges, A.; Fornaro, A.; Freitas, H.; Moreira, A.; Andrade, M. F.; Environ. Monit. Assess. 2009, 149, 241.

2. Manahan, S. E.; Environmental Chemistry, $8^{\text {th }}$ ed., CRC Press: Boca Raton, 2005.

3. Nriagu, J.; Pacyna, J.; Nature 1988, 333, 134.

4. Miranda, R. M.; Andrade, M. F.; Fattori, A. P.; Atmos. Res. 2005, 75, 135; Miranda, R. M; Andrade, M. F.; Worobiec, A.; Grieken, R. V.; Atmos. Environ. 2002, 36, 345.

5. Paoliello, M. M. B.; De Capitani, E. M. Em Metais: Gerenciamento da Toxicidade; Azevedo, F., ed.; Ed. Atheneu: São Paulo, 2003.

6. Jambers, W.; Dekov, V.; Van Grieken, R.; Sci. Total Environ. 2000, 256, 133.

7. Davidson, C. I.; Wu, Y. Em Control and fate of atmospheric Trace Metals; Pacyna, J.; Ottar, B., eds.; Kluwer Academic Publishers: 
Holanda, NATO ASI Series, Series C: Mathematical and Physical Sciences, 268, 1988, p. 147.

8. Buat-Ménard, P. Em Global Atmospheric Chemical Change; Hewitt, C. N.; Sturges, W. T., eds.; Chapman \& Hall: Londres, 1995.

9. Hu, G. P.; Balasubramaniam, R.; Water, Air, Soil Pollut. 2003, 144, 285.

10. Morselli, L.; Olivieri, P.; Brusori, B.; Passarini, F.; Environ. Pollut. 2003, $124,457$.

11. Fornaro, A.; Gutz, I. G. R.; Atmos. Environ. 2006, 40, 5893.

12. Leal, T. M. F.; Fontenele, A. P. G.; Pedrotti, J. J.; Fornaro, A.; Quim. Nova 2004, 27, 855; Santos, M. A.; Illanes, C. A. F.; Fornaro, A.; Pedrotti, J. J.; Water, Air, Soil Pollut. Focus 2007, 7, 85.

13. Fornaro, A.; Gutz, I. G. R.; Atmos. Environ. 2003, 37, 117.

14. Santos, M. A.; Damasceno, T. V. F.; Fornaro, A.; Pedrotti, J. J.; Resumos da 29a. Reunião Anual da Sociedade Brasileira de Química, Águas de Lindóia, Brasil, 2006.

15. Santos, M. A.; Pedrotti, J. J.; Fornaro, A. Em Highway and Urban Environment, Proceedings of the $8^{\text {th }}$ Highway and Urban Environment Symposium, Series: Alliance for Global Sustainability Bookseries; Gregory, M. M.; Sébastien, R., eds.; Dordrecht: Países Baixos, 2007, vol. 12 , cap. 2.

16. Richter, E. M.; Pedrotti, J. J.; Angnes, L.; Electroanalysis 2003, 15, 23.

17. Pedrotti, J. J.; Angnes, L.; Gutz, I. G. R.; Anal. Chim. Acta 1994, 298 , 393.

18. Pedrotti, J. J.; Gutz, I. G. R.; Talanta 2003, 60, 695.

19. Miller, J. C.; Miller, J. N.; Statistics for Analytical Chemistry, $2^{\text {nd }}$ ed. John Wiley \& Sons: New York, 1988.

20. Roux, M. Em Applied Multivariate Analysis in SAR and Environmental Studies; Devillers, J.; Karcher, W., eds.; Kluwer Academic Publishers: Holanda, 1991

21. Garnaud, S.; Mouchel, J. M.; Chebbo, G.; Thévenot, D. R.; Sci. Total Environ. 1999, 235, 235.
22. Wang, Y.; Huang, K.; Li, C.; Mi, H.; Luo, J.;Tsai, P.; Atmos. Environ. 2003, 37, 4637.

23. Grömping, A. H. J.; Ostapulck, P.; Emons, H.; Chemosphere 1997, 34, 2227.

24. Halstead, M. J. R.; Cunninghame, R. G.; Hunter, K. A.; Atmos. Environ. 2000, 34, 665.

25. Al-Momani, I. F.; Atmos. Environ. 2003, 37, 4507.

26. Pike, S. M.; Moran, S. B.; Atmos. Environ. 2001, 35, 3361.

27. Kanenolloupolou, E. A.; Global Nest: the International Journal 2001, 3 , 45, http://www.gnest.org/Journal/Vol3_No1/kanellopoulou.pdf, acessada em Julho 2008.

28. Viel, F.; Dissertação de Mestrado, Universidade Federal de Santa Catarina, Brasil, 2002.

29. Migliavacca, D.; Teixeira, E. C.; Pires, M.; Fachel, J.; Atmos. Environ 2004, 38, 1641; Migliavacca, D. M.; Teixeira, E. C.; Wiegand, F.; Machado, A. C. M.; Sanchez, J.; Atmos. Environ. 2005, 39, 1829; Braga, C. F.; Teixeira, E. C.; Migliavacca, D.; Carvalho, F. G.; Quim. Nova 2002, 25, 92; Migliavacca, D. M.; Teixeira, E. C.; Machado, A. C. M.; Quim. Nova 2005, 28, 371.

30. Galloway, J. N.; Dentener, F. J.; Capone, D. G.; Boyer, E. W.; Howarth, R. W.; Seitzinger, S. P.; Asner, G. P.; Cleveland, C. C.; Green, P. A.; Holland, E. A.; Karl, D. M.; Michaels, A. F.; Porter, J. H.; Townsend, A. R.; Vorosmarty, C. J.; Biogeochemistry 2004, 70, 153; Elliott, S.; Simpson, I. J.; Blake, D. R.; Bossert, J. E.; Chow, J.; Colina, J. A.; Dubey, M. K.; Duce, R. A.; Edgerton, S.; Gaffney, J.; Gupta, M.; Guzman, F.; Matson, P. A.; McNair, L. A.; Ortiz, E.; Riley, W.; Rowland, F. S.; Ruiz, M. E.; Russell, A. G.; Smith, F. A.; Sosa, G.; Streit, G.; Watson, J.; Environ. Sci. Policy 2000, 3, 145.

31. Heeb, N. V.; Forss, A. M.; Brühlmann, S.; Lüscher, R.; Saxer, C. J.; Hug, P.; Atmos. Environ. 2006, 40, 5986.

32. Ozaki, H.; Watanabe, I.; Kuno, K.; Water Air Soil Pollut. 2004, 157, 209. 